Tôhoku Math. Journ.

25 (1973), 1-22.

\title{
LOCAL PROPERTY OF THE SINGULAR SETS OF SOME KLEINIAN GROUPS
}

\author{
TOHRU AKAZA*)
}

(Received April 1, 1972)

Introduction. In the recent paper [3], I proved the existence of Kleinian groups with fundamental domains bounded by four circles whose singular sets have positive 1-dimensional measure. Now in the natural way the following problem arises; to what extent does the Hausdorff dimension of the singular sets of Kleinian groups climb up, when the number $N$ of the boundary circles increases? It is conjectured and seems still open that the 2-dimensional measure of the singular sets $E$ of general finitely generated Kleinian groups is always zero (see [1]).

The purpose of this paper is to investigate the properties of computing functions introduced in No. 3 of $\S 1$ in detail and the local property of the singular set of the Kleinian group by using these properties.

We shall state preliminaries and notations about Kleinian groups in $\S 1$. We shall prove the main theorem giving the relation between the computing function and the Hausdorff measure of the singular set of Kleinian group in $\S 2$. At last in $\S 3$ we shall seek for the relation between the computing function and the Hausdorff dimension of the singular set and further give an application to the convergence problem of Poincaré theta-series by using the main theorem.

\section{§1. Preliminaries and Notations.}

1. Let us denote by $B$ the domain bounded by $N$ mutually disjoint circles $H_{i}, H_{i}^{\prime}(1 \leqq i \leqq p)$ and $K_{j}(1 \leqq j \leqq q)$ and form the properly discontinuous group of linear transformations with the fundamental domain $B$, where $N=2 p+q$.

Let $S_{i}$ be a hyperbolic or loxodromic generator which transforms the outside of $H_{i}$ onto the inside of $H_{i}^{\prime}$. Then $\left\{S_{i}\right\}_{i=1}^{p}$ generates a Schottky group whose fundamental domain is bounded by $\left\{H_{i}, H_{i}^{\prime}\right\}_{i=1}^{p}$. Let $\left\{S_{j}^{*}\right\}_{j=1}^{q}$ be the elliptic transformations with period 2 corresponding to $\left\{K_{j}\right\}_{j=1}^{q}$. Then $\left\{S_{j}^{*}\right\}_{j=1}^{q}$ generates a properly discontinuous group whose fundamental domain is the outside of the boundary circles $\left\{K_{j}\right\}_{j=1}^{q}$.

*) This work was supported in part by a research contract AF 49 (638)-1591 (1967-68). 
By combining two groups, a new group $G$, which is generated by $\left\{S_{i}\right\}_{i=1}^{p}$ and $\left\{S_{j}^{*}\right\}_{j=1}^{q}$, is obtained and is a kind of Kleinian groups. We often use the notation $\mathscr{Y}$ to denote the set of $\left\{S_{i}\right\}_{i=1}^{p}$, their inverses and $\left\{S_{j}^{*}\right\}_{j=1}^{q}$. It is easily seen that the fundamental domain of $G$ coincides with $B$. We denote the singular set of $G$ by $E$.

Defining the product $S T$ in $G$ by $S T(z)=S(T(z))$, we can write any element of $G$ in the form

$$
S=S_{\left(\nu_{k}\right)} S_{j_{k}}^{*} \cdots S_{\left(\nu_{1}\right)} S_{j_{1}}^{*} S_{\left(\nu_{0}\right)},
$$

where the indices $\nu_{i}(i=0, \cdots, k)$ are non-negative integers and $S_{\left(\nu_{i}\right)}$ denotes the product of $\nu_{i}$ generators of the Schottky group or their inverses and $S_{j_{i}}^{*}$ denotes any element of $\left\{S_{j}^{*}\right\}_{j=1}^{q}$. We call the sum

$$
m=\sum_{i=0}^{k} \nu_{i}+k
$$

the grade of $S$ and for simplicity we use the notation $S_{(m)}$ to clarify the grade $m$ of $S$.

The image $S_{(m)}(B)$ of the fundamental domain $B$ by $S_{(m)}(\in G)$ with grade $m(\geqq 1)$ is bounded by $N$ circles

$$
S_{(m)}\left(H_{i}\right), S_{(m)}\left(H_{i}^{\prime}\right) \text {, and } S_{(m)}\left(K_{j}\right) \quad(i=1, \cdots, p ; j=1, \cdots, q)
$$

and for simplicity we call the outer boundary circles $C$ of $S_{(m)}(B)$ a circle of grade $m$. The number of circles of grade $m$ is obviously equal to $N(N-1)^{m-1}$.

Consider two arbitrary transformations $T$ and $S$ of $G$. We assume that $S \neq T^{-1}$, where $T^{-1}$ denotes the inverse of $T$. Denote by $I_{S}, I_{T^{-1}}$ and $I_{S T}$ the isometric circles of $S, T^{-1}$ and $S T$, respectively. Let $R_{S}, R_{T}$ and $R_{S T}$ be radii of $I_{S}, I_{T}$ and $I_{S T}$, respectively. As to these values, the relation

$$
R_{S T}=\frac{R_{S} \cdot R_{T}}{\left|T(\infty)-S^{-1}(\infty)\right|}
$$

holds. The isometric circle of a transformation with grade $m$ is called the isometric circle of grade $m$.

2. Denoting by $r$ and $r_{i}(i=1, \cdots, N-1)$ the radius of the outer boundary circle $C$ and the radii of $N-1$ inner boundary circles $C_{i}$ $(i=1, \cdots, N-1)$ of the image $S(B)(S \in G)$, we have the following two propositions ([2]).

Proposition 1. There exist positive constants $K_{0}(<1)$ and $k_{0}$ depending only on $B$ such that 


$$
k_{0} r \leqq r_{i} \leqq K_{0} r \quad(i=1, \cdots, N-1) .
$$

Proposition 2. There exist positive constants $k(G, \mu)$ and $K(G, \mu)$ depending on $G$ and $\mu$ such that

$$
k(G, \mu)\left(R_{S}\right)^{\mu} \leqq r^{\mu / 2} \leqq K(G, \mu)\left(R_{S}\right)^{\mu},
$$

where $\mu$ is any positive number.

Denote by $F_{n_{0}}$ the family of all closed discs bounded by circles of grade $n\left(\geqq n_{0}\right)$. It is easily seen that $F_{n_{0}}$ is a covering of the singular set of our Kleinian group $G$ and by Proposition 1 we see that the diameter of any disc of $F_{n_{0}}$ is less than a given $\delta(>0)$ for a sufficiently large integer $n_{0}$.

For such a covering $F_{n_{0}}$ we have the following important proposition ([2]).

Proposition 3. Let $F_{n_{0}}^{o / k_{0}}$ be a covering of $E$ constructed by discs in $F_{n_{0}}$ whose radii are not greater than $\delta /\left(2 k_{0}\right)$ and let $r_{c}$ be the radius of $a$ disc $C$ in $F_{n_{0}}^{\text {ol }}$, where $k_{0}$ is a positive constant in Proposition 1 . Then it holds

$$
L_{\eta}(E)=\lim _{\delta \rightarrow 0} \inf _{\left\{F_{n_{0}}^{\delta / k_{0}}\right\}} \sum_{\mathcal{L} \in F_{n_{0}}^{\delta / k_{0}}}\left(2 r_{c}\right)^{\eta} \leqq \mathscr{K}\left(k_{0} / 2\right)^{-\eta} M_{\eta}(E),
$$

where $\mathscr{X}$ is an absolute constant and $M_{\eta}(E)$ denotes the $\eta$-dimensional measure of $E$.

3. Let $T$ be any fixed element of $\mathscr{Y}$. Denote by $H_{T}$ and $H_{T^{-1}}$ the boundary circles of $B$ which are equivalent by $T$, that is, $H_{T}=T\left(H_{T^{-1}}\right)$ and further by $D_{T}$ the closed disc bounded by $H_{T}$. If $H_{T}$ is one of $K_{j}(1 \leqq j \leqq q)$, then $H_{T^{-1}}=H_{T}$.

Let $S_{(n)}=T_{n} T_{n-1} \cdots T_{2} T_{1}\left(T_{i} \in \mathscr{Y}\right)$ be any element of $G$ with grade $n$ and be the following form:

$$
S_{(n)}(z)=\frac{a z+b}{c z+d}, \quad a d-b c=1 .
$$

If we take the derivative of $S_{(n)}(z)$, we obtain easily

$$
\left|\frac{d S_{(n)}(z)}{d z}\right|^{\mu / 2}=\left(\frac{1}{|c z+d|}\right)^{\mu}=\left(\frac{R_{S_{(n)}}}{\left|S_{(n)}^{-1}(\infty)-z\right|}\right)^{\mu}, \quad 0<\mu<4,
$$

where $S_{(n)}^{-1}$ denotes the inverse $\left(S_{(n)}\right)^{-1}=T_{1}^{-1} \cdots T_{n}^{-1}$ of $S_{(n)}$.

Forming the sum of $(N-1)^{n}$ terms with respect to all $S_{(n)}$ such that $T_{1} \neq T^{-1}$ and $T_{i} \neq T_{i+1}^{-1}(1 \leqq i \leqq n-1)$, we have the following function

$$
\sum_{S_{(n)}}\left(\frac{R_{S_{(n)}}}{\left|S_{(n)}^{-1}(\infty)-z\right|}\right)^{\mu}=\sum_{S_{(n)}}\left|\frac{d S_{(n)}(z)}{d z}\right|^{\mu / 2}, \quad\left(T_{1} \neq T^{-1}\right) .
$$


The domain of definition of this function is $D_{T}$. We denote it by $\chi_{n}^{(\mu ; T)}(z)$.

Since $z$ moves on $D_{T}$ and $T_{1} \neq T^{-1}$, the $(N-1)^{n}$ denominators of (1.7) do not vanish, and hence $\chi_{n}^{(\mu ; T)}(z)$ is uniformly continuous in $D_{T}$. Let $S_{(m)}$ be an element of the form $S_{(m)}=T S_{(m-1)}$. Using the relations (1.1) and (1.7), we obtain easily

$$
\chi_{n}^{(\mu ; T)}\left(S_{(m)}(\infty)\right)=\sum_{S_{(n)}}\left(\frac{R_{S_{(n)}}}{\left|S_{(n)}^{-1}(\infty)-S_{(m)}(\infty)\right|}\right)^{\mu}=\sum_{S_{(n)}}\left(\frac{R_{S_{(n)} S_{(m)}}}{R_{S_{(m)}}}\right)^{\mu},
$$

where $S_{(n)} S_{(m)}=S_{(n+m)}$.

We call $\chi_{n}^{(\mu ; T)}(z)$ the $\mu$-dimensional computing function of order $n$ on $T$ and there exist $N$ computing functions $\chi_{n}^{(\mu ; T)}(z)$ corresponding to the choice of $T$ from $\mathscr{Y}$.

If we differentiate $S_{(n)}(z)=T_{n} T_{n-1} \cdots T_{1}(z)$ with respect to $z$, we get

$$
\left|\frac{d S_{(n)}(z)}{d z}\right|^{\mu / 2}=\prod_{i=1}^{n}\left|\frac{d T_{i}\left(z_{i}\right)}{d z_{i}}\right|^{\mu / 2}, \quad z_{i}=T_{i-1} \cdots T_{1}(z), \quad z \in D_{T},
$$

where $z_{1}=z$. Hence $\chi_{n}^{(\mu ; T)}(z)$ is also written in the following form

$$
\chi_{n}^{(\mu: T)}(z)=\sum_{S_{(n)}}\left(\prod_{i=1}^{n}\left|\frac{d T_{i}\left(z_{i}\right)}{d z_{i}}\right|^{\mu / 2}\right)
$$

and this representation coincides with the function

$$
f_{T}^{(\mu)} n(z)=\sum_{S_{(n)}}\left\{\prod_{i=1}^{n}\left(\frac{R_{T_{i}}}{\left|T_{i}^{-1}(\infty)-T_{i-1} \cdots T_{1}(z)\right|}\right)^{\mu}\right\}, \quad 0<\mu<4, z \in D_{T},
$$

which was already introduced in ([3]).

By using $\chi_{n}^{(\mu ; T)}(z)$ we have the following proposition.

Proposition 4 ([3]). Let $G$ be a Kleinian group as in No. 1. If there exist some positive integer $n_{0}$ and a constant $\sigma$ such that

$$
\chi_{n_{0}}^{(\mu ; T)}(z)>\sigma>1,
$$

on the subset $E \cap D_{T}$ for any $T \in \mathscr{V}$, then $M_{\mu / 2}(E)$ is positive.

4. Now let us seek for the relations between two computing functions on the different elements of $\mathscr{V}$ and between two computing functions on the same $T$ with different orders.

Proposition 5. It holds the following relation between two computing functions on the different elements of $\mathscr{Y}$ :

$$
K(\mu) \sum_{S_{(l)}} \chi_{n}^{\left(\mu ; T_{l}\right)}\left(S_{(l)}(z)\right)>\chi_{n+l}^{(\mu ; T)}(z)>k(l, \mu) \sum_{S_{(l)}} \chi_{n}^{\left(\mu ; T_{l}\right)}\left(S_{(l)}(z)\right),
$$

where $K(\mu)$ is a constant depending only on $\mu$, but $k(l, \mu)$ is a constant 
depending on $l$ and $\mu$ and tends to zero for $l \rightarrow \infty$. Further it holds the following relation between two computing functions on the same $T$ with different orders:

$$
K_{1}(n, \mu) \chi_{l}^{(\mu ; T)}(z)>\chi_{n+l}^{(\mu ; T)}(z)>K_{0}(n, \mu) \chi_{i}^{(\mu ; T)}(z),
$$

where $K_{i}(n, \mu)(i=0,1)$ denote the constants depending only on $n$ and $\mu$.

Proof. Fix an element $T \in \mathscr{Y}$ and take a transformation $S_{(n+l)}=$ $S_{(n)} S_{(l)}=T_{n+l} T_{n+l-1} \cdots T_{l+1} T_{l} \cdots T_{2} T_{1}$ with grade $n+l$ such that $T_{1} \neq T^{-1}$, where $T$ and $T_{j} \in \mathscr{V}(1 \leqq j \leqq n+l)$. If we differentiate the transformation $S_{(n+l)}(z)=S_{(n)} S_{(l)}(z)\left(z \in D_{T}\right)$ with respect to $z$, we have

$$
\left|\frac{d S_{(n+l)}(z)}{d z}\right|^{\mu / 2}=\left(\left|\frac{d S_{(n)}\left(z^{\prime}\right)}{d z^{\prime}}\right| \cdot\left|\frac{d S_{(l)}(z)}{d z}\right|\right)^{\mu / 2}, \quad z^{\prime}=S_{(l)}(z) \text {. }
$$

Hence we get from (1.6)

$$
\left(\frac{R_{S_{(n+l)}}}{\left|S_{(n+l)}^{-1}(\infty)-z\right|}\right)^{\mu}=\left(\frac{R_{S_{(n)}}}{\left|S_{(n)}^{-1}(\infty)-S_{(l)}(z)\right|} \cdot \frac{R_{S_{(l)}}}{\left|S_{(l)}^{-1}(\infty)-z\right|}\right)^{\mu} .
$$

Forming the sum of $(N-1)^{n+l}$ terms with respect to all $S_{(n+l)}$ of grade $n+l$ in $G$ such that $T_{1} \neq T^{-1}$, we obtain from the definition of the computing function the following relation

$$
\chi_{n+l}^{(\mu ; T)}(z)=\sum_{S_{(l)}}\left\{\chi_{n}^{\left(\mu ; T_{l}\right)}\left(S_{(l)}(z)\right)\left(\frac{R_{S_{(l)}}}{\left|S_{(l)}^{-1}(\infty)-z\right|}\right)^{\mu}\right\}
$$

where the domains of definition of $\chi_{n+l}^{(\mu ; T)}(z)$ and $\chi_{n}^{\left(\mu ; T_{l}\right)}(z)$ are the closed discs $D_{T}$ and $D_{T_{l}}$ bounded by $H_{T}$ and $H_{T_{l}}$, respectively.

Since $S_{(l)}^{-1}(\infty)=T_{1}^{-1} \cdots T_{l}^{-1}(\infty)$ and $z$ are contained in $D_{T_{1}^{-1}}$ and $D_{T}$, respectively and $T \neq T_{1}^{-1}$, each denominator of the right hand side in (1.15) does not vanish and is greater than some positive constant from the assumption about $B$. Noting that $R_{S_{(l)}}$ tends to zero for $l \rightarrow \infty$, we have the above inequality (1.11).

Since $S_{(n)}^{-1}(\infty)$ and $S_{(l)}(z)$ are contained in $D_{T_{l+1}^{-1}}$ and $D_{T_{l}}$ in (1.14), respectively, the factor $\left|S_{(n)}^{-1}(\infty)-S_{(l)}(z)\right|$ is greater than some positive constant from $T_{l} \neq T_{l+1}^{-1}$. Noting the definition of the computing function, we can easily get from (1.14) the above inequality.

5. Now let us give an important property of the computing function.

THEOREM 1. (i) Suppose that the sequence of computing functions $\left\{\chi_{n}^{(\mu ; T)}(z)\right\}(n=1,2, \cdots)$ on some $T \in \mathscr{Y}$ is bounded at some point $z_{0} \in E \cap D_{T}$. Then $\left\{\chi_{n}^{(\mu ; T)}(z)\right\}(n=1,2, \cdots)$ is uniformly bounded and equi-continuous on $D_{T}$. 
(ii) Suppose that the sequence of computing functions $\left\{\chi_{n}^{(\mu ; T)}(z)\right\}$ $(n=1,2, \cdots)$ on some $T \in \mathscr{Y}$ diverges for $n \rightarrow \infty$ at some point $z_{0} \in E \cap D_{T}$. Put $\eta_{n}^{(\mu ; T)}(z)=1 / \chi_{n}^{(\mu ; T)}(z)$. Then $\left\{\eta_{n}^{(\mu ; T)}(z)\right\}(n=1,2, \cdots)$ is uniformly bounded and equi-continuous on $D_{T}$.

Proof. (i) From the definition of the computing function we have for any $z \in D_{T}$ the following estimation:

$$
\left|\chi_{n}^{(\mu ; T)}(z)-\chi_{n}^{(\mu ; T)}\left(z_{0}\right)\right| \leqq \sum_{S_{n}} R_{S_{(n)}^{\mu}}^{\mu} \frac{|| S_{(n)}^{-1}(\infty)-\left.z_{0}\right|^{\mu}-\left|S_{(n)}^{-1}(\infty)-z\right|^{\mu} \mid}{\left|S_{(n)}^{-1}(\infty)-z\right|^{\mu}\left|S_{(n)}^{-1}(\infty)-z_{0}\right|^{\mu}}
$$

Let us consider the behavior of the function $\left|S_{(n)}^{-1}(\infty)-z\right|^{\mu}(0<\mu<4)$ in $D_{T}$. Since $S_{(n)}^{-1}(\infty)=T_{1}^{-1} \cdots T_{n}^{-1}(\infty)$ and $z$ are contained in $D_{T_{1}^{-1}}$ and $D_{T}$, respectively, and $T \neq T_{1}^{-1}$, there is a positive constant $\rho$ depending only on $B$ such that

$$
\left|S_{(n)}^{-1}(\infty)-z\right|^{\mu}>\rho \text {. }
$$

Denoting the rectangular coordinates $S_{(n)}^{-1}(\infty), z$ and $z_{0}$ by $\left(a_{n}, b_{n}\right),(x, y)$ and $\left(x_{0}, y_{0}\right)$, respectively, we can represent this function in the following form:

$$
\left|S_{(n)}^{-1}(\infty)-z\right|^{\mu}=\left\{\left(x-a_{n}\right)^{2}+\left(y-b_{n}\right)^{2}\right\}^{\mu / 2}, \quad 0<\mu<4 .
$$

The partial derivatives of $\left|S_{(n)}^{-1}(\infty)-z\right|^{\mu}$ with respect to $x$ and $y$ are $\mu\left|S_{(n)}^{-1}(\infty)-z\right|^{\mu-2}\left(x-a_{n}\right)$ and $\mu\left|S_{(n)}^{-1}(\infty)-z\right|^{\mu-2}\left(y-b_{n}\right)$, respectively and both functions are continuous on $D_{T}$ from the assumption on $B$. Using the mean value theorem to $\left|S_{(n)}^{-1}(\infty)-z\right|^{\mu}$, we obtain easily

$$
|| S_{(n)}^{-1}(\infty)-\left.z\right|^{\mu}-\left|S_{(n)}^{-1}(\infty)-z_{0}\right|^{\mu}\left|\leqq \mathscr{K}^{\prime}\right| z-z_{0} \mid,
$$

where $\mathscr{K}$ is a constant depending only on $B$. Thus we have from (1.16), (1.17) and (1.19)

$$
\left|\chi_{n}^{(\mu ; T)}(z)-\chi_{n}^{(\mu ; T)}\left(z_{0}\right)\right| \leqq \frac{\mathscr{K}\left|z-z_{0}\right|}{\rho} \chi_{n}^{(\mu ; T)}\left(z_{0}\right) .
$$

Denote by $d$ the maximum among the values of the diameters of $N$ boundary circles $\left\{H_{i}, H_{i}^{\prime}\right\}_{i=1}^{p} \cup\left\{K_{j}\right\}_{j=1}^{q}$. Then we have from (1.20)

$$
\chi_{n}^{(\mu ; T)}(z) \leqq K \chi_{n}^{(\mu ; T)}\left(z_{0}\right),
$$

where $K=(\mathscr{K} \cdot d / \rho)+1$ is a constant depending only on $B$. Since (1.20) is symmetric with respect to any pair of points $z$ and $z_{0}$ contained in $D_{T}$, we have

$$
\frac{1}{K} \chi_{n}^{(\mu ; T)}\left(z_{0}\right) \leqq \chi_{n}^{(\mu ; T)}(z) \leqq K \chi_{n}^{(\mu ; T)}\left(z_{0}\right)
$$


which shows that $\left\{\chi_{n}^{(\mu ; T)}(z)\right\} \quad(n=1,2, \cdots)$ is uniformly bounded on $D_{T}$ under the assumption of boundedness of $\left\{\chi_{n}^{(\mu ; T)}\left(z_{0}\right)\right\}(n=1,2, \cdots)$.

Take any two points $z$ and $z^{\prime}$ in $D_{T}$. Since $\chi_{n}^{(\mu ; T)}(z)<M$ on $D_{T}$ from the fact proved above, we have from (1.20)

$$
\left|\chi_{n}^{(\mu ; T)}\left(z^{\prime}\right)-\chi_{n}^{(\mu ; T)}(z)\right|<\frac{\mathscr{K} \cdot M}{\rho}\left|z^{\prime}-z\right| \cdot
$$

Thus $\left\{\chi_{n}^{(\mu ; T)}(z)\right\}(n=1,2, \cdots)$ is equi-continuous on $D_{T}$.

(ii) Since $\lim _{n \rightarrow \infty} \chi_{n}^{(\mu ; T)}\left(z_{0}\right)=\infty,\left\{\eta_{n}^{(\mu ; T)}\left(z_{0}\right)\right\}(n=1,2, \cdots)$ is bounded at $z_{0}$. We have the following estimation:

$$
\begin{aligned}
& \left|\eta_{n}^{(\mu ; T)}(z)-\eta_{n}^{(\mu ; T)}\left(z_{0}\right)\right| \\
& \leqq \\
& \quad \eta_{n}^{(\mu ; T)}\left(z_{0}\right) \eta_{n}^{(\mu ; T)}(z)\left|\sum_{S_{(n)}} R_{S_{(n)}}^{\mu}\left(\frac{1}{\left|S_{(n)}^{-1}(\infty)-z_{0}\right|^{\mu}}-\frac{1}{\left|S_{(n)}^{-1}(\infty)-z\right|^{\mu}}\right)\right| \\
& =\eta_{n}^{(\mu ; T)}\left(z_{0}\right) \eta_{n}^{(\mu ; T)}(z) \mid \sum_{S_{(n)}} \frac{R_{S_{(n)}}^{\mu}\left|S_{(n)}^{-1}(\infty)-z\right|^{\mu}}{\left|S_{(n)}^{-1}(\infty)-z_{0}\right|^{\mu}} \\
& \quad \times\left(\frac{\left|S_{(n)}^{-1}(\infty)-z\right|^{\mu}-\left|S_{(n)}^{-1}(\infty)-z_{0}\right|^{\mu}}{\mid-1}\right) \mid
\end{aligned}
$$

In the same manner and notations as in (i), we get the following estimation:

$$
\left|\eta_{n}^{(\mu ; T)}(z)-\eta_{n}^{(\mu ; T)}\left(z_{0}\right)\right| \leqq \frac{\mathscr{K}\left|z-z_{0}\right|}{\rho} \eta_{n}^{(\mu ; T)}\left(z_{0}\right)
$$

Denoting by $d$ the maximum among the values of the diameters of $N$ boundary circles $\left\{H_{i}, H_{i}^{\prime}\right\}_{i=1}^{p} \cup\left\{K_{j}\right\}_{j=1}^{q}$ as in (i), we have also from (1.25)

$$
\frac{1}{K} \eta_{n}^{(\mu ; T)}\left(z_{0}\right) \leqq \eta_{n}^{(\mu ; T)}(z) \leqq K \eta_{n}^{(\mu ; T)}\left(z_{0}\right)
$$

where $K=(\mathscr{K} \cdot d / \rho)+1$ is the same constant as in (1.21). Hence we can show that $\left\{\eta_{n}^{(\mu ; T)}(z)\right\}(n=1,2, \cdots)$ is uniformly bounded on $D_{T}$ under the assumption at $z_{0}$. The proof of the equi-continuity of $\left\{\eta_{n}^{(\mu ; T)}(z)\right\}(n=1,2, \cdots)$ is also the same manner as in (i).

q.e.d.

\section{$\S 2$. Local property of the computing function.}

6. By using the condition (1.10) (for $\mu=2$ ) of Proposition 4, the existence of a Kleinian group with the fundamental domain bounded by four circles, whose singular set has positive 1-dimensional measure, was shown in [3]. On the other hand, it is well known that the 2-dimensional measure of the singular sets of the Kleinian groups defined in No. 1 is always zero. Therefore it seems an important and interesting problem 
to decide the upper bound of the value of the dimension for which the singular set of our Kleinian group has positive measure, though our Kleinian group is somewhat special as a Kleinian group. For this purpose we must investigate profoundly the complicated property of the singular set, in particular, the local property of it. We shall find that the computing function gives the efficient tool to investigate the property of the singular set of our Kleinian group.

7. Now let us give the main theorem under the preliminaries of the computing function in $\$ 1$.

THEOREM 2. The following three propositions are equivalent to each other: (1) The sequence $\left\{\chi_{n}^{\left(\mu ; T^{*}\right)}(z)\right\}(n=1,2, \cdots)$ on some fixed $T^{*}(\in \mathscr{Y})$ diverges (or converges to zero) at some singular point $z_{0} \in E \cap D_{T^{*}}$, that is,

$$
\lim _{n \rightarrow \infty} \chi_{n}^{\left(\mu ; T^{*}\right)}\left(z_{0}\right)=\infty \quad(\text { or } 0) \text { for some } z_{0} \in E \cap D_{T^{*}}
$$

(2) It holds

$$
\lim _{n \rightarrow \infty} \chi_{n}^{(\mu ; T)}(z)=\infty \quad(\text { or } 0)
$$

for any $T(\in \mathscr{Y})$ uniformly on $D_{T}$.

(3) $M_{\mu / 2}(E)=\infty($ or 0$)$.

As the proof of this theorem is complicated, we shall divide it into six lemmas. At first we shall prove that (1) is equivalent to (2). Since (1) is valid under (2), it is sufficient to show only that (1) implies (2).

Now we shall give the following lemma.

LEMma 1. Suppose that the proposition (1) of Theorem 2 holds. Then it holds

$$
\lim _{n \rightarrow \infty} \chi_{n}^{(\mu ; T)}(z)=\infty \quad(\text { or } 0)
$$

for any $T(\in \mathscr{Y})$ uniformly on $D_{T}$.

Proof. (i) The case of the limit 0.

Take any point $z \in D_{T^{*}}$. Then we have from (1.22)

$$
\frac{1}{K} \chi_{n}^{\left(\mu ; T^{*}\right)}\left(z_{0}\right) \leqq \chi_{n}^{\left(\mu ; T^{*}\right)}(z) \leqq K \chi_{n}^{\left(\mu ; T^{*}\right)}\left(z_{0}\right)
$$

We can determine for any $\varepsilon$ the order $n_{0}\left(T^{*}\right)$ depending on $\varepsilon, T^{*}$ and $z_{0}$ such that

$$
\chi_{n}^{\left(\mu ; T^{*}\right)}\left(z_{0}\right)<\frac{\varepsilon}{K}
$$


for any $n\left(\geqq n_{0}\right)$. We obtain from $(2.3)$

$$
\chi_{n}^{\left(\mu ; T^{*}\right)}<\varepsilon, \text { for any } z \in D_{T^{*}} .
$$

Now from (1.11) of Proposition 5 we have for $z_{0}\left(\in E \cap D_{T^{*}}\right)$ and its image $T\left(z_{0}\right)\left(\in E \cap D_{T}, T \in \mathscr{Y}\right)$ the following inequality

$$
\chi_{n+1}^{(\mu ; T)}\left(z_{0}\right)>k(1, \mu) \chi_{n}^{(\mu ; T)}\left(T\left(z_{0}\right)\right) .
$$

Since $\lim _{n \rightarrow \infty} \chi_{n}^{\left(\mu ; F^{*}\right)}\left(z_{0}\right)=0$ from the assumption, it holds for $T\left(z_{0}\right)$

$$
\lim _{n \rightarrow \infty} \chi_{n}^{(\mu ; T)}\left(T\left(z_{0}\right)\right)=0 \text {. }
$$

Hence from the above method there exists the order $n_{0}(T)$ depending on $\varepsilon, T$ and $T\left(z_{0}\right)$ such that it holds for any $n\left(\geqq n_{0}(T)\right)$ and for any $z \in D_{T}$

$$
\chi_{n}^{(\mu ; T)}(z)<\varepsilon .
$$

If we denote $\max _{T \in \vartheta} n_{0}(T)$ by $n^{*}$, we see easily that it holds for any $T$ and any $n\left(\geqq n^{*}\right)$

$$
\chi_{n}^{(\mu ; T)}(z)<\varepsilon
$$

on $D_{T}$. Thus it holds

$$
\lim _{n \rightarrow \infty} \chi_{n}^{(\mu ; T)}(z)=0
$$

for any $T(\in \mathscr{Y})$ uniformly on $D_{T}$.

(ii) The case of the limit $\infty$.

Take any point $z \in D_{T^{*}}$. Then we have from (1.26)

$$
\frac{1}{K} \eta_{n}^{\left(\mu ; T^{*}\right)}\left(z_{0}\right) \leqq \eta_{n}^{\left(\mu ; T^{*}\right)}(z) \leqq K \eta_{n}^{\left(\mu ; T^{*}\right)}\left(z_{0}\right) .
$$

Let $n_{0}\left(T^{*}\right)$ be the order depending on $\varepsilon, T^{*}$ and $z_{0}$ such that it holds $\eta_{n}^{\left(\mu ; T^{*}\right)}\left(z_{0}\right)<\varepsilon / K$ for any $n\left(\geqq n_{0}\right)$. Then we obtain from (2.9)

$$
\eta_{n}^{\left(\mu ; T^{*}\right)}(z)<\varepsilon \quad \text { for any } z \in D_{T^{*}} .
$$

From (1.11) of Proposition 5 we have for any $z^{\prime}\left(\in E \cap D_{T}\right)$ and its image $T^{*}\left(z^{\prime}\right)\left(\in E \cap D_{T^{*}}\right)$ the inequality

$$
\eta_{n+1}^{(\mu ; T)}\left(z^{\prime}\right)<\frac{1}{K(1, \mu)} \eta_{n}^{\left(\mu ; T^{*}\right)}\left(T^{*}\left(z^{\prime}\right)\right) .
$$

Since $\lim _{n \rightarrow \infty} \eta_{n}^{(\mu ; * *)}\left(T^{*}\left(z^{\prime}\right)\right)=0$ from $(2.10)$, it holds

$$
\lim _{n \rightarrow \infty} \eta_{n+1}^{(\mu ; T)}\left(z^{\prime}\right)=0 \text {. }
$$

Hence from the method in (i) there exists the order $n_{0}(T)$ depending on $\varepsilon, T$ and $z^{\prime}$ such that it holds for any $n\left(\geqq n_{0}(T)\right)$ and any $z \in D_{T}$ 


$$
\eta_{n}^{(\mu ; T)}(z)<\varepsilon \text {. }
$$

If we denote $\max _{T \in \mathcal{Y}} n_{0}(T)$ by $n^{*}$, we see easily that it holds for any $T$ and any $n\left(\geqq n^{*}\right)$

$$
\eta_{n}^{(\mu ; T)}(z)<\varepsilon
$$

on $D_{T}$. Thus it holds

$$
\lim _{n \rightarrow \infty} \chi_{n}^{(\mu ; T)}(z)=\infty
$$

for any $T(\in \mathscr{Y})$ uniformly on $D_{T}$.

q.e.d.

Thus we could prove that, in Theorem 2, (1) is equivalent to (2).

8. Suppose that the sequence $\left\{\chi_{n}^{\left(\mu ; T^{*}\right)}(z)\right\}(n=1,2, \cdots)$ diverges to infinity (or converges to zero) at some point $z_{0} \in E \cap D_{T^{*}}$ for a fixed $T^{*}(\in \mathscr{Y})$. Then any subsequence $\left\{\chi_{n_{i}}^{\left(\mu ; T^{*}\right)}(z)\right\}(i=1,2, \cdots)$ diverges to infinity (or converges to zero) at $z_{0}$. Conversely, we shall prove the following result.

Lemma 2. Suppose that for some subsequence $\left\{\chi_{n_{i}}^{\left(\mu ; T^{*}\right)}(z)\right\}(i=1,2, \cdots)$ of $\left\{\chi_{n}^{\left(\mu ; F^{*}\right)}(z)\right\}(n=1,2, \cdots)$ with respect to some $T^{*}(\in \mathscr{Y})$

$$
\lim _{i \rightarrow \infty} \chi_{n_{i}}^{\left(\mu ; T^{*}\right)}\left(z_{0}\right)=\infty \quad(\text { or } 0) \text { at some } z_{0} \in E \cap D_{T^{*}} .
$$

Then it holds

$$
\lim _{n \rightarrow \infty} \chi_{n}^{\left(\mu ; T^{*}\right)}\left(z_{0}\right)=\infty \quad(\text { or } 0) .
$$

Proof. (i) At first we shall prove the case for $\infty$. If we replace the sequence $\left\{\chi_{n}^{\left(\mu ; 7^{*}\right)}(z)\right\} \quad(n=1,2, \cdots)$ with the subsequence $\left\{\chi_{n_{i}}^{\left(\mu ; *^{*}\right)}(z)\right\}$ $(i=1,2, \cdots)$ in Proposition (1) of Theorem 2, we obtain from Proposition (2) of it that it holds

$$
\lim _{n \rightarrow \infty} \chi_{n_{i}}^{(\mu / T)}(z)=\infty
$$

uniformly on $D_{T}$ for any $T(\in \mathscr{Y})$. Then for any large number $M$ there exists some positive integer $n_{0}$ depending only on $M$ such that it holds for any $z \in D_{T}$

$$
\chi_{n_{0}}^{(\mu ; T)}(z)>M .
$$

Consider the computing function $\chi_{q_{0}}^{\left(\mu ; T^{*}\right)}(z)$ at $z_{0}$, where $q$ is a positive integer. Then for any small $\varepsilon^{\prime}(>0)$ there exists some positive number $\delta^{\prime}\left(\varepsilon^{\prime}\right)$ depending only on $\varepsilon^{\prime}$ such that

$$
\chi_{q n_{0}}^{\left(\mu ; T^{*}\right)}\left(z_{0}\right)>\chi_{q n_{0}}^{\left(\mu ; T^{*}\right)}(z)-\varepsilon^{\prime}, \quad \text { for any } z \in D_{\delta^{\prime}}\left(z_{0}\right) \cap D_{T^{*}}
$$

where $D_{\delta^{\prime}}\left(z_{0}\right)$ denotes a disc of radius $\delta^{\prime}$ with center $z_{0}$. Hence if we take 
a sufficiently large integer $l$, then there exists an $S_{(l)} \in G$ such that $S_{(l)}(\infty)$ is contained in $D_{\delta^{\prime}}\left(z_{0}\right)$ and such that

$$
\chi_{q n_{0}}^{\left(\mu ; T^{*}\right)}\left(z_{0}\right)>\chi_{q n_{0}}^{\left(\mu ; T^{*}\right)}\left(S_{(l)}(\infty)\right)-\varepsilon^{\prime} .
$$

Here we have from (1.8)

$$
\chi_{q n_{0}}^{\left(\mu ;: *^{*}\right)}\left(S_{(l)}(\infty)\right)=\frac{\sum_{S_{\left(q n_{0}\right)}}\left(R_{\left.S_{\left(q n_{0}\right.}\right)_{s(l)}}\right)^{\mu}}{\left(R_{\left.S_{(l)}\right)^{\mu}}\right)^{\mu}} .
$$

We can modify the right hand side of (2.20) as in the following:

$$
\frac{\sum_{\left(q n_{0}\right)}\left(R_{\left.S_{\left(q n_{0}\right.}\right)_{(l)}}\right)^{\mu}}{\left(R_{S_{(l)}}\right)^{\mu}}=\prod_{j=1}^{q}\left[\frac{\sum_{s_{\left(j n_{0}\right)}}\left(R_{S_{\left(j n_{0}\right)} s_{(l)}}\right)^{\mu}}{\sum_{S_{\left((j-1) n_{0}\right)}}\left(R_{\left.\left.S_{\left((j-1) n_{0}\right.}\right)_{(l)}\right)^{\mu}}^{\mu}\right.}\right],
$$

where $S_{\left.((0)) n_{0}\right)}$ is the identity. Since

$$
\frac{\sum_{S_{\left(n_{0}\right)}}\left(R_{S_{\left(j n_{0}\right)} S_{(l)}}\right)^{\mu}}{\left(R_{\left.S_{\left((j-1) n_{0}\right)} S_{(l)}\right)^{\mu}}^{\mu}\right.}=\chi_{n_{0}}^{\left(\mu ; T_{i}\right)}\left(S_{\left((j-1) n_{0}\right)} S_{(l)}(\infty)\right), \quad(j \geqq 1),
$$

we have from $(2.18)$

$$
\chi_{n_{0}}^{\left(\mu ; T_{i}\right)}\left(S_{\left((j-1) n_{0}\right)} S_{(l)}(\infty)\right)>M,
$$

where $S_{\left((j-1) n_{0}\right)} S_{(l)}=T_{i} S_{\left((j-1) n_{0}+l-1\right)}$ and $T_{i} \in \mathscr{Y}$. Applying (2.22) to (2.21), we obtain from (2.20)

$$
\chi_{q n_{0}}^{\left(\mu ; T^{*}\right)}\left(S_{(l)}(\infty)\right)>M^{q}
$$

and we get from (2.19)

$$
\chi_{q n_{0}}^{\left(\mu ; T^{*}\right)}\left(z_{0}\right)>M^{q}-\varepsilon^{\prime} .
$$

Since $M$ can be taken sufficiently large, $\chi_{q n_{0}}^{\left(\mu ; T^{*}\right)}\left(z_{0}\right)$ tends to $\infty$ for $q \rightarrow \infty$. Thus we find that under the assumption (2.15) there exists a subsequence $\left\{\chi_{q n_{0}}^{\left(\mu ; T^{*}\right)}(z)\right\}(q=1,2, \cdots)$ such that it holds

$$
\lim _{q \rightarrow \infty} \chi_{q n_{0}}^{\left(\mu ; * T^{*}\right)}\left(z_{0}\right)=\infty \text {. }
$$

Let $\chi_{n^{\prime}}^{\left(\mu ; T^{*}\right)}(z)$ be any term of $\left\{\chi_{n}^{\left(\mu ; T^{*}\right)}(z)\right\}(n=1,2, \cdots)$. Then we have from (1.12)

$$
K_{1}\left(n_{0}, \mu\right) \chi_{g n_{0}}^{\left(\mu ; T^{*}\right)}\left(z_{0}\right)>\chi_{n^{\prime}}^{\left(\mu ; T^{*}\right)}\left(z_{0}\right)>K_{0}\left(n_{0}, \mu\right) \chi_{g n_{0}}^{\left(\mu ; ; * \pi_{0}^{*}\right)}\left(z_{0}\right),
$$

where $n^{\prime}=q n_{0}+n^{*}\left(n^{*}<n_{0}\right)$. Therefore we obtain

$$
\lim _{n \rightarrow \infty} \chi_{n}^{\left(\mu ; T^{*}\right)}\left(z_{0}\right)=\infty \text {. }
$$

(ii) Next we shall treat the case for zero. 
If we replace the sequence $\left\{\chi_{n}^{\left(\mu ; T^{*}\right)}(z)\right\}(n=1,2, \cdots)$ with the subsequence $\left\{\chi_{n_{i}}^{\left(\mu ; T^{*}\right)}(z)\right\}(i=1,2, \cdots)$ in Proposition (1) of Theorem 2, we obtain from Proposition (2) of it that there exists for any small number $\varepsilon(<1)$ some positive integer $n_{0}$ depending only on $\varepsilon$ such that it holds

$$
\chi_{n_{0}}^{(\mu ; T)}(z)<\varepsilon,
$$

for any $T(\in \mathscr{Y})$ and any $z \in D_{T}$. In the analogous way as in (i), we can easily prove from $(2.21)$ that for $\left\{\chi_{n}^{\left(\mu ; r^{*}\right)}(z)\right\}(n=1,2, \cdots)$ it holds

$$
\lim _{n \rightarrow \infty} \chi_{n}^{\left(\mu ; T^{*}\right)}\left(z_{0}\right)=0 \text {. }
$$

q.e.d.

9. Now let us prove that (2) is equivalent to (3) in Theorem 2. Since the proof is complicated, we divide it into four lemmas.

Suppose that $\lim _{n \rightarrow \infty} \chi_{n}^{(\mu ; T)}(z)=\infty$ for any $T(\in \mathscr{Y})$ uniformly on $D_{T}$. Then for any large number $M$ there exists some positive integer $n_{0}$ such that it holds

$$
\chi_{\left.n_{0} ; T\right)}^{(\mu)}(z)>M
$$

for any $T(\in \mathscr{Y})$ and any $z \in D_{T}$.

From (1.8) it holds

$$
\chi_{n_{0}}^{(\mu ; T)}\left(S_{\left(l_{0}\right)}(\infty)\right)=\sum_{S_{\left(n_{0}\right)}}\left(\frac{R_{S_{\left(n_{0}\right)} S_{\left(l_{0}\right)}}}{R_{S_{\left(l_{0}\right)}}}\right)^{\mu}>M .
$$

Since

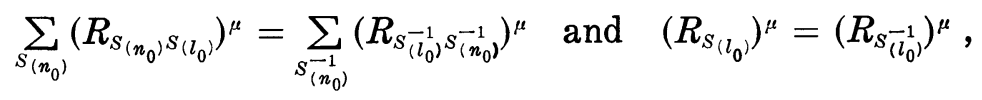

we get

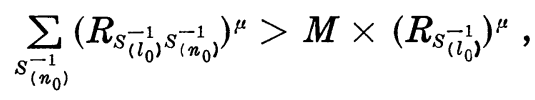

where $\sum_{S_{\left(n_{0}\right)}^{-1}}$ denotes the sum of the radii of isometric circles $I_{S_{\left(l_{0}\right)}^{-1}} S_{\left(n_{0}\right)}^{-1}$ when $S_{\left(n_{0}\right)}^{-1}$ runs over all the transformations with grade $n_{0}$ whose left elements are not equal to the inverse of the right element of $S_{\left(l_{0}\right)}^{-1}$.

This was the sufficient condition for the ( $/ / 2)$-dimensional measure of $E$ to be positive and was given in [3]. Thus we have the following lemma.

Lemma 3 ([3]). Suppose that $\lim _{n \rightarrow \infty} \chi_{n}^{(\mu ; T)}(z)=\infty$ for any $T(\in \mathscr{Y})$ uniformly on $D_{T}$. Then it holds $M_{\mu / 2}(E)>0$.

10. On the other hand we can easily prove the following lemma.

LEMMA 4. Suppose that $\lim _{n \rightarrow \infty} \chi_{n}^{(\mu ; T)}(z)=0$ for any $T(\in \mathscr{Y})$ uniformly on $D_{T}$. Then it holds $M_{\mu / 2}(E)=0$. 
Proof. We can find, from the assumption, some positive integer $n_{0}$ depending only on any given small $\varepsilon$ such that it holds

$$
\chi_{n_{0}}^{(\mu ; T)}(z)<\varepsilon
$$

for any $T(\in \mathscr{Y})$ and any $z \in D_{T}$. Consider the image of the infinity $S_{(l)}(\infty)\left(S_{(l)}=T S_{(l-1)}\right)$ by $S_{(l)}(z)$. Then we have from (2.30) the following inequality:

$$
\chi_{n_{0}}^{(\mu ; T)}\left(S_{(l)}(\infty)\right)=\sum_{S_{\left(n_{0}\right)}}\left(\frac{R_{S_{\left(n_{0}\right)} S_{(l)}}}{R_{S_{(l)}}}\right)^{\mu}=\sum_{S_{\left(n_{0}\right)}^{-1}}\left(\frac{R_{\left.S^{-1}\right)}^{-1} S_{\left(n_{0}\right)}^{-1}}{R_{S_{(l)}^{-1}}^{-1}}\right)^{\mu}<\varepsilon .
$$

Denote by $r_{S_{(i)}^{(i)}}\left(i=1, \cdots, N(N-1)^{l-1}\right)$ the radii of circles $C_{S_{(i)}^{(i)}}$ of grade $l$ greater than $n_{0}$. Then from (1.3) of Proposition 2, we get the following inequality

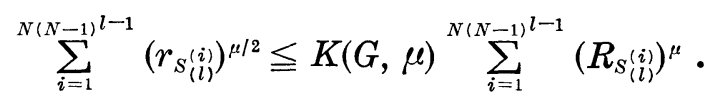

Putting $l=p \cdot n_{0}+q_{0}\left(1 \leqq q_{0}<n_{0}\right)$, where $p$ is a positive integer and arranging $(N-1)^{n_{0}}$ circles $N-1$ by $N-1$ with respect to all inner boundary circles contained in all circles of grade $n_{0}$, we obtain from (2.31)

$$
\begin{aligned}
& \sum_{i=1}^{N(N-1) l-1}\left(R _ { S _ { ( l ) } ^ { ( i ) } ) ^ { \mu } < ( \varepsilon ) ^ { p } } \sum _ { j = 1 } ^ { N ( N - 1 ) q _ { 0 } - 1 } \left(R_{\left.S_{\left(q_{0}\right)}^{(j)}\right)^{\mu}}\right.\right. \\
& \leqq(\varepsilon)^{p} \max _{1 \leqq \nu q_{0}}\left(\sum_{j=1}^{N(N-1))^{\nu-1}}\left(R_{\left.S_{(\nu)}^{(j)}\right)^{\mu}}\right) .\right.
\end{aligned}
$$

Since the right hand side of (2.33) tends to 0 for $p \rightarrow \infty$, we obtain from (2.32)

$$
\lim _{l \rightarrow \infty} \sum_{i=1}^{N(N-1) l-1}\left(r_{\left.S_{(l)}^{(i)}\right)^{\mu / 2}}=0 .\right.
$$

Thus we can conclude that $M_{\mu / 2}(E)=0$ under the assumption of Lemma. q.e.d.

11. Now let us prove the following lemma.

LEMma 5. The following two propositions are equivalent to each

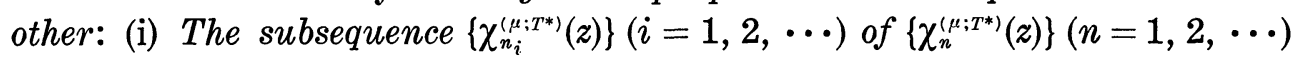
on some fixed $T^{*}(\in \mathscr{Y})$ converges to $\alpha(\neq 0)$ at some $z_{0} \in E \cap D_{T^{*}}$ (ii)

$$
0<M_{\mu / 2}(E)<\infty \text {. }
$$

Proof. We have already showed in Lemma 1 that the propositions (1) and (2) in Theorem 2 are equivalent to each other. From this result and Lemma 2 we can easily see that the above proposition (i) is equivalent to 


$$
0<\underline{\chi}^{(\mu ; T)}(z) \leqq \bar{\chi}^{(\mu ; T)}(z)<\infty,
$$

for any $T(\in \mathscr{Y})$ and any $z \in D_{T}$, where

$$
\underline{\chi}^{(\mu ; T)}(z)=\varliminf_{n \rightarrow \infty} \chi_{n}^{(\mu ; T)}(z) \quad \text { and } \quad \bar{\chi}^{(\mu ; T)}(z)=\varlimsup_{n \rightarrow \infty} \chi_{n}^{(\mu ; T)}(z) .
$$

Hence it is sufficient to show that (2.34) is equivalent to (2.35). We divide the proof into two parts (A) and (B).

12. (A). At first we shall prove that $\bar{\chi}^{(\mu ; T)}(z)<\infty$ implies $M_{\mu / 2}(E)<\infty$ and that $0<M_{\mu / 2}(E)$ implies $\underline{\chi}^{(\mu ; T)}(z)>0$. Suppose that $\bar{\chi}^{(\mu ; T)}(z)<\infty$ establishes for any $T(\in \mathscr{Y})$. Then from Theorem $1\left\{\chi_{n}^{(\mu ; T)}(z)\right\} \quad(n=1,2, \cdots)$ is uniformly bounded. Hence $\max _{T \in \mathcal{Y}}\left(\sup _{z \in D_{T}} \bar{\chi}^{(\mu ; T)}(z)\right)=M$ is a finite number, that is,

$$
\chi_{n}^{(\mu ; T)}(z) \leqq M
$$

for any $n, T(\in \mathscr{Y})$ and $z\left(\in D_{T}\right)$.

Take any large integer $l_{0}$ and consider all closed discs

$$
D_{S_{\left(l_{0}\right)}^{(j)}}\left(j=1, \cdots, N(N-1)^{l_{0}-1}\right)
$$

bounded by all circles of grade $l_{0}$.

Take any closed disc $D_{S_{\left(l_{0}\right)}}$ bounded by $C_{S_{\left(l_{0}\right)}}$ among the above discs for such a fixed $l_{0}$. Denote by $r_{s_{(l)}^{(i)}}\left(i=1, \cdots,(N-1)^{\left.l-l_{0}\right)}\right.$ the radii of the inner boundary circles $C_{S_{(l)}^{(i)}}$ of grade $l\left(>l_{0}\right)$ contained in $C_{S_{\left(l_{0}\right)}}$. Then from (1.3) of Proposition 2, we get the inequality

$$
\sum_{i=1}^{(N-1) l-l_{0}}\left(r_{S_{(l)}^{(i)}}\right)^{\mu / 2} \leqq K(G, \mu) \sum_{i=1}^{(N-1) l-l_{0}}\left(R_{\left.S_{(l)}^{(i)}\right)^{\mu}},\right.
$$

where $S_{(l)}^{(i)}=S_{\left(l_{0}\right)} S_{\left(l-l_{0}\right)}^{(i)}$. We can modify the sum of the right hand side of (2.37) in the following:

$$
\begin{aligned}
\sum_{i=1}^{(N-1) l-l_{0}}\left(R_{S_{(l)}^{(i)}}\right)^{\mu} & =\sum_{i=1}^{(N-1) l-l_{0}}\left(\frac{R_{\left.\left(S_{\left(l-l_{0}\right)}^{(i)}\right)^{-1} S_{\left(l_{0}\right)}^{-1}\right)}}{R_{S_{\left(l_{0}\right)}^{-1}}}\right)^{\mu} \times\left(R_{\left.S_{\left(l_{0}\right.}^{-1}\right)^{\mu}}\right. \\
& =\chi_{l-l_{0}}^{(\mu ; T)}\left(S_{\left(l_{0}\right)}^{-1}(\infty)\right) \times\left(R_{\left.S_{\left(l_{0}\right.}^{-1}\right)^{\mu}},\right.
\end{aligned}
$$

where $S_{\left(l_{0}\right)}^{-1}=T S_{\left(l_{0}-1\right)}$. Hence we have from (2.36), (2.37) and (2.38)

$$
\sum_{i=1}^{(N-1) l-l_{0}}\left(r_{s_{(l)}^{(i)}}\right)^{\mu / 2} \leqq K(G, \mu)\left(R_{\left.S_{\left(l_{0}\right)}^{-1}\right)^{\mu}} M\right. \text {. }
$$

Since $D_{S_{(i)}^{(i)}}\left(i=1, \cdots,(N-1)^{\left.l-l_{0}\right)}\right.$ is a covering of $E \cap D_{S_{\left(l_{0}\right)},}$, we obtain from

$$
M_{\mu / 2}\left(E \cap D_{S_{\left(l_{0}\right)}}\right)<+\infty .
$$

The above inequality (2.39) holds for any closed disc bounded by the 
circle of grade $l_{0}$ and hence we can conclude that $M_{\mu / 2}(E)<\infty$ under the assumption (2.35). We see easily from (2.37) and (2.38)

$$
M_{\mu / 2}\left(E \cap D_{S_{\left(l_{0}\right)}}\right) \leqq K(G, \mu) \chi_{l-l_{0}}^{(\mu ; T)}\left(S_{\left(l_{0}\right)}^{-1}(\infty)\right) \times\left(R_{S_{\left(l_{0}\right)}^{-1}}\right)^{\mu} .
$$

If we suppose that $M_{\mu / 2}(E)>0$, there is at least one closed disc $D_{S_{\left(l_{0}\right)}}$ among all closed disc $D_{S_{\left(l_{0}\right)}^{(j)}}\left(j=1, \cdots, N(N-1)^{l_{0}-1}\right)$ such that

$$
M_{\mu / 2}\left(E \cap D_{\left.S_{\left(l_{0}\right)}\right)}>0\right. \text {. }
$$

Hence we have from Theorem 1 and (2.41) that $M_{\mu / 2}(E)>0$ implies that $\underline{\chi}^{(\mu ; T)}(z)>0$ for any $T(\in \mathscr{Y})$ and any $z \in D_{T}$.

13. (B). Next we shall prove that $M_{\mu / 2}(E)<\infty$ implies $\bar{\chi}^{(\mu ; T)}(z)<\infty$ for any $T(\in \mathscr{Y})$ and any $z \in D_{T}$ and that $\underline{\chi}^{(\mu ; T)}(z)>0$ implies $M_{\mu / 2}(E)>0$.

Assume that this proposition is not true. From Lemmas 1 and 2 it is easy to see that the sequence $\left\{\chi_{n}^{(\mu ; T)}(z)\right\}(n=1,2, \cdots)$ diverges for any $T(\in \mathscr{Y})$ uniformly on $D_{T}$. Hence there exists some positive integer $l_{0}$ depending on any positive number $M$ such that it holds for any $T$

$$
\chi_{l_{0}}^{(\mu ; T)}(z)>M
$$

on $D_{T}$.

Consider all closed dises $D_{s_{\left(l_{1}\right)}^{(j)}}\left(j=1, \cdots, N(N-1)^{l_{1}-1}\right)$ bounded by . $C_{S_{\left(l_{1}\right)}^{(j)}}$ with grade $l_{1}\left(>l_{0}\right)$. Take any closed disc $D_{S_{\left(l_{1}\right)}}$ bounded by $C_{S_{\left(l_{1}\right)}}$ from these discs. Let $F_{n_{0}^{\prime}}^{\delta / k_{0}}$ be a covering of $E$ defined in Proposition 3

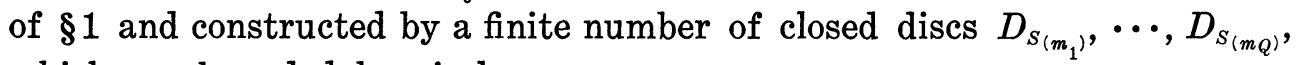
which are bounded by circles

$$
C_{S_{\left(m_{1}\right)}}, \cdots, C_{S_{\left(m_{Q}\right)}},
$$

respectively, where $C_{S_{\left(m_{j}\right)}}(1 \leqq j \leqq Q)$ is a circle of grade $m_{j}$. Here we assume that $\delta$ is a small number such that $m_{j}$ is sufficiently large and satisfies the condition

$$
m_{j}-l_{1}>l_{0}
$$

Let us denote by

$$
C_{S_{\left(n_{1}\right)}, \cdots, C_{S_{\left(n_{R}\right)}}, \quad(R<Q)}
$$

the circles among (2.43) contained in $C_{S_{\left(l_{1}\right)}}$ as the inner boundary circles.

Denote $\min _{1 \leqq j \leqq R}\left(n_{j}\right)$ by $n^{*}$. We amend the circles (2.45) in the following: (i) if $n_{j}-n^{*}$ is an integral multiple of $l_{0}$, we leave the circle $C_{S_{\left(n_{j}\right)}}$, and (ii) if $n_{j}-n^{*}=l_{0} \cdot p+q\left(0<q<l_{0}\right)$, where $p$ is a positive integer, we replace the circle $C_{S_{\left(n_{j}\right)}}$ with the $(N-1)^{l_{0}-q}$ circles

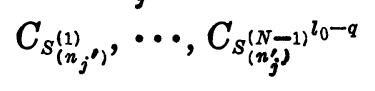


of grade $n_{j^{\prime}}$ contained in $C_{S_{\left(n_{j}\right)}}$, where $n_{j}^{\prime}-n^{*}=l_{0}(p+1)$. After such amendment we get a new subcovering of $E \cap D_{S_{\left(l_{1}\right)}}$, whose elements are all discs bounded by the circles of grade $n^{*}+l_{0} \cdot p$. Denote such circles by

$$
C_{S_{\left(n_{1}^{\prime}\right)}}, C_{S_{\left(n_{2}^{\prime}\right)}}, \cdots, C_{S_{\left(n_{U}^{\prime}\right)}}, \quad(R \leqq U) .
$$

Then we get from (1.2) of Proposition 1 the following inequality:

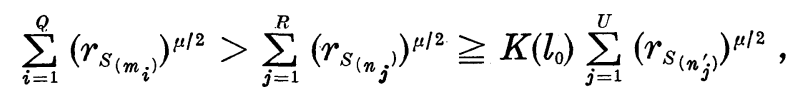

where $r_{S_{\left(m_{i}\right)},}, r_{S_{\left(n_{j}\right)}}$ and $r_{S_{\left(n_{j}^{\prime}\right)}}$ denote the radii of the circles (2.43), (2.45) and (2.46), respectively, and $K\left(l_{0}\right)$ is the constant depending only on $l_{0}$ and $B$. By using (1.3) of Proposition 2, we obtain

$$
\sum_{j=1}^{U}\left(r_{S_{\left(n_{j}^{\prime}\right)^{\prime}}}\right)^{\mu / 2} \geqq K(G, \mu) \sum_{j=1}^{U}\left(R_{S_{\left(n_{j}^{\prime}\right)^{\prime}}}\right)^{\mu} .
$$

In the set of circles (2.46) there exist a finite number of systems $W_{n_{k}^{*}}$ $(k=1, \cdots, n)$, each of which consists of $(N-1)^{l_{0}}$ boundary circles with the following properties: (i) $(N-1)^{l_{0}}$ circles of each $W_{n_{k}^{*}}$ have the same grade number $n_{k}^{*}$, while the grades of circles of different systems are not necessarily equal, (ii) $(N-1)^{l_{0}}$ circles of each system $W_{n_{k}^{*}}$ are the totality of inner boundary circles which are contained in a circle of grade $n_{k}^{*}-l_{0}$.

These $(N-1)^{l_{0}}$ circles in each $W_{n_{k}^{*}}$ are arranged $N-1$ by $N-1$ and are replaced by circles of grade $n_{k}^{*}-1$ and after that, we repeat also such procedure and so on. After $l_{0}$ times of such procedure, we reach to the circle of grade $n_{k}^{*}-l_{0}$, that is, the outer boundary circle of $S_{\left(n_{k}^{*}-l_{0}\right)}(B)$. If $M>1$ is supposed in (2.42), it holds for each system

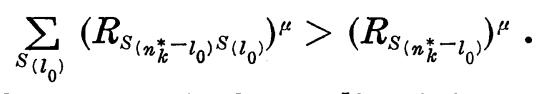

Here $\sum_{s_{\left(l_{0}\right)}}$ denotes the sum of the radii of isometric circles $I_{\left.S_{\left(n_{b}^{*}-l_{0}\right)} S_{\left(l_{0}\right)}\right)}$, when $S_{\left(l_{0}\right)}$ runs over all the transformations with grade $l_{0}$ whose left elements are not equal to the inverse of the right element of $S_{\left(n_{k}^{*}-l_{0}\right)}$. After replacing $(N-1)^{l_{0}}$ circles of each system $W_{n_{k}^{*}}$ by an outer boundary circle of $S_{\left(n_{k}^{*}-l_{0}\right)}(B)$, we have also a new covering of $E \cap D_{S_{\left(l_{1}\right)}}$ consisting of closed discs which are denoted by

$$
D_{S_{\left(n_{1}^{\prime \prime}\right)}}, D_{S_{\left(n_{2}^{\prime}\right)}}, \cdots, D_{S_{\left(n_{V}^{\prime \prime}\right)}}, \quad(V<U) .
$$

Repeating the above procedure to these circles and continuing $(p-1)$ times, we obtain the following inequality

$$
\sum_{j=1}^{L}\left(R_{\left.S_{\left(n_{j}^{\prime}\right.}\right)^{\mu}} \geqq \sum_{S_{\left(n^{*}-l_{1}\right)}}\left(R_{\left.S_{\left(n^{*}\right)}\right)^{\mu}}, S_{\left(n^{*}\right)}=S_{\left(l_{1}\right)} S_{\left(n^{*}-l_{1}\right)},\right.\right.
$$

where the summation in the right hand side is taken over all transforma- 
tions in $G$ with grade $n^{*}$ such that the images $S_{\left(n^{*}\right)}(B)$ are contained in $D_{S_{\left(l_{1}\right)}}$.

Since

$$
\sum_{s_{\left(n^{*}-l_{1}\right)}}\left(R_{S_{\left(l_{1}\right)} S_{\left(n^{*}-l_{1}\right)}}\right)^{\mu}=\sum_{S_{\left(n^{*}-l_{1}\right)}^{-1}}\left(R_{\left.\left.S_{\left(n^{*}-l_{1}\right.}^{-1}\right)_{\left(l_{1}\right)}^{-1}\right)^{\mu}}\right.
$$

we have

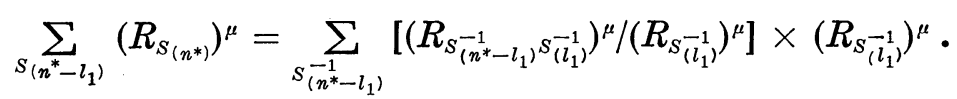

We see easily from (1.8) that the term in the bracket of the right hand

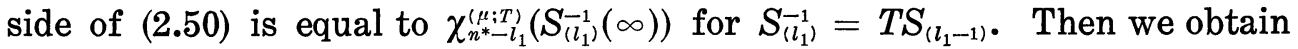
from (2.42) and (2.44)

$$
\chi_{n^{*}-l_{1}}^{(\mu ;)}\left(S_{\left(l_{1}\right)}^{-1}(\infty)\right)>M
$$

Hence we have from (2.50)

$$
\sum_{S_{\left(n^{*}-l_{1}\right)}}\left(R_{S_{\left(n^{*}\right)}}\right)^{\mu}>\left(S_{\left.S_{\left(l_{1}\right.}^{-1}\right)^{\mu}} \times M .\right.
$$

Since (2.51) holds for any closed disc $D_{S_{\left(l_{1}\right)}}$, we obtain from (1.4), (2.47), (2.48), (2.49) and (2.51) the following inequality:

$$
\begin{aligned}
& \mathscr{K}\left(\frac{1 k_{0}}{2}\right)^{-\mu / 2} M_{\mu / 2}\left(E \cap D_{T}\right) \\
& \quad \geqq K\left(l_{0}\right) K(G, \mu)\left(\sum_{S_{\left(l_{1}\right)}}\left(R_{\left.S_{\left(l_{1}\right.}^{-1}\right)^{\mu}}\right)^{\mu}\left(R_{T}^{-1}\right)^{\mu}\right) \times\left(R_{T}^{-1}\right)^{\mu} \times M .
\end{aligned}
$$

Here we have already assumed that $M$ is any positive number and $l_{1}$ is any fixed positive integer greater that $l_{0}$. If we take a sufficiently large number $l_{1}$ for such a fixed $l_{0}$, we see easily that (2.52) leads to the contradiction under $M_{\mu / 2}(E)<\infty$. Thus we could prove that it holds $\bar{\chi}^{(\mu ; T)}(z)<\infty$ for any $T(\in \mathscr{Y})$ and any $z \in D_{T}$ under the assumption $M_{\mu / 2}(E)<\infty$.

Let us prove that $M_{\mu / 2}(E)>0$ holds under the assumption that $\underline{\chi}^{(\mu ; T)}(z)>0$ for any $T(\in \mathscr{Y})$ and any $z \in D_{T}$.

We obtain easily from (2.47), (2.48) and (2.50)

$$
\sum_{j=1}^{R}\left(r_{S_{\left(n_{j}\right.}}\right)^{\mu / 2} \geqq K\left(l_{0}\right) K(G, \mu)\left(R_{\left.S_{\left(l_{1}\right.}^{-1}\right)^{\mu}} \chi_{n^{*} \neq-l_{1}}^{(\mu) T}\left(S_{\left(l_{1}\right)}^{-1}(\infty)\right) .\right.
$$

If $\delta$ tends to 0 in $F_{n}^{\delta / / k_{0}}$, the radii of the inner boundary circles of (2.45) contained in $C_{S\left(l_{1}\right)}$ tend also to 0 and hence from (2.48) and (2.49) the grade number $n^{*}$ tends to $\infty$. Therefore we have from (1.4) and (2.53)

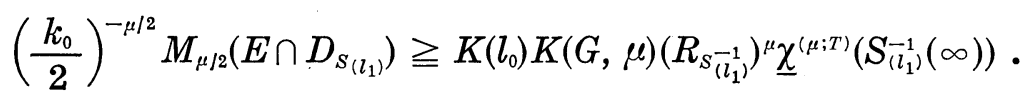


If $M_{\mu / 2}(E)=0$ is assumed, it is easily concluded that $\underline{\chi}^{(\mu ; T)}\left(S_{\left(l_{1}\right)}^{-1}(\infty)\right)=0$. Hence from Lemmas 1 and 2 it holds for any $T(\in \mathscr{Y})$

$$
\underline{\chi}^{(\mu ; T)}(z)=\lim _{n \rightarrow \infty} \chi_{n}^{(\mu ; T)}(z)=0
$$

uniformly on $D_{T}$.

Thus we obtain from the contrapositive proposition that $\underline{\chi}^{(\mu ; T)}(z)>0$ implies that $M_{\mu / 2}(E)>0$.

q.e.d.

14. Now let us prove that (2) is equivalent to (3) in Theorem 2.

LEMma 6. If $M_{\mu / 2}(E)=0$, then it holds that $\lim _{n \rightarrow \infty} \chi_{n}^{(\mu ; T)}(z)=0$ for any $T(\in \mathscr{Y})$ uniformly on $D_{T}$.

Proof. From Lemmas 1 and 2 , it is sufficient to show that $\underline{\chi}^{(\mu ; T)}\left(z_{0}\right)=0$ for some $T$ and for some $z_{0} \in D_{T}$. If $0<\underline{\chi}^{(\mu ; T)}\left(z_{0}\right)$ for some $\left.\overline{T(} \in \mathscr{Y}\right)$ and some point $z_{0} \in D_{T}$, we have from Lemma 5 that $0<M_{\mu / 2}(E)$, which is also a contradiction.

q.e.d.

Now we can give the proof of Theorem 2. We can conclude from Lemmas 4 and 6 that $M_{\mu / 2}(E)=0$ is equivalent to the proposition that it holds $\lim _{n \rightarrow \infty} \chi_{n}^{(\mu ; T)}(z)=0$ uniformly on $D_{T}$ for any $T(\in \mathscr{Y})$. Therefore we get also from Lemmas 1,2 and 5 the following result: $M_{\mu / 2}(E)=\infty$ is equivalent to the proposition that it holds $\lim _{n \rightarrow \infty} \chi_{n}^{(\mu ; T)}(z)=\infty$ uniformly on $D_{T}$ for any $T(\in \mathscr{Y})$. Thus Theorem 2 was completely proved. q.e.d.

Arranging the above result, we have from Theorem 2 and Lemma 2 the following Theorem.

THEOREm 3. In Proposition (1) of Theorem 2 the assumption for $\left\{\chi_{n}^{(\mu ; T)}(z)\right\}(n=1,2, \cdots)$ can be replaced with that for the subsequence $\left\{\chi_{n_{i}}^{\left(\prime \prime \prime T^{*}\right)}(z)\right\}(i=1,2, \cdots)$.

\section{§3. Hausdorff dimension of the singular set of a Kleinian group.}

15. Let us investigate the relation between the computing function and Hausdorff dimension of the singular set of a Kleinian group. Given a compact set $F$ in the $z$-plane, the Hausdorff dimension of $F$ is the unique non-negative number $d(F)$ satisfying

$$
M_{d}(F)=0, \quad \text { if } \quad d>d(F)
$$

and

$$
M_{d}(F)=+\infty \text {, if } 0 \leqq d<d(F),
$$

where $M_{d}(F)$ denotes the $d$-dimensional Hausdorff measure of $F$.

The following is well-known ([4]). 
Proposition 6. Let $F$ be any point set in the z-plane and suppose that $\alpha>0$. If $M_{\alpha}(F)<+\infty$ and $\alpha<\beta$, then $M_{\beta}(F)=0$.

16. Looking at the above definition of the Hausdorff dimension of a point set and considering Theorem 2 and Proposition 6, we may define the Hausdorff dimension of the singular set $E$ of a Kleinian group $G$ in the following way.

DeFinition. Let $T$ be any generator or its inverse of the Kleinian group $G$, that is, let $T \in \mathscr{Y}$. Then the Hausdorff dimension $d(E)$ of the singular set $E$ of $G$ is defined as

$$
\begin{aligned}
& \sup \left\{\frac{\mu}{2}: \lim _{n \rightarrow \infty} \chi_{n}^{(n ; T)}(z)=\infty, \text { for some } T \text { and for some } z \in D_{T}\right\} \\
= & \inf \left\{\frac{\mu^{\prime}}{2}: \lim _{n \rightarrow \infty} \chi_{n}^{\left(\mu^{\prime \prime} ; T\right)}(z)=0, \text { for some } T \text { and for some } z \in D_{T}\right\} .
\end{aligned}
$$

We shall prove the following theorem.

THEOREM 4. Let $d(E)=\mu_{0} / 2$ be the Hausdorff dimension of $E$. Then $M_{\mu_{0} / 2}(E)$ is positive and finite.

RRoof. If $M_{\mu_{0} / 2}(E)=0$ is assumed, then for any sufficiently small $\varepsilon$ there exists some positive integer $n_{0}$ such that

$$
\chi_{n_{0}}^{\left(\mu_{0}: T\right)}(z)<\varepsilon
$$

for any $T \in \mathscr{Y}$ and any $z \in D_{T}$ from Theorem 2. Since $\chi_{n_{0}}^{(\mu ; T)}(z)$ is a continuous function of $\mu$ for a fixed $n_{0}$ and any $z$, we can take a positive number $\delta$ depending only on $\varepsilon$ such that

$$
\chi_{n_{0}}^{\left(\mu_{0}-\hat{\delta} ; T\right)}(z)<2 \varepsilon .
$$

Then we have also from the method of Lemma 4 that $M_{\left(\mu_{0}-\tilde{\partial}\right) / 2}(E)=0$. This contradicts the assumption that $\mu_{0} / 2$ is the Hausdorff dimension.

Next if $M_{\mu_{0} / 2}(E)=\infty$ is assumed, we get also a contradiction in the similar manner. Thus we can see that $M_{\mu_{0} / 2}(E)$ is finite. q.e.d.

From Theorems 2, 3 and the above theorem we have the following corollary.

CoRollary. Let $d(E)=\mu_{0} / 2$ be the Hausdorff dimension of $E$. Then $\bar{\chi}^{\left(\mu_{0}: T\right)}(z)$ and $\underline{\chi}^{\left(\mu_{0}: T\right)}(z)$ for any $T(\in \mathscr{Y})$ are both positive and finite on $D_{T}$.

It is natural that the following problem arises in the case of the finite limit of the sequence of computing functions. Let $\mu_{0} / 2$ be the Hausdorff dimension of $E$. Does $\bar{\chi}^{\left(\mu_{0} ; T\right)}(z)$ equal $\underline{\chi}^{\left(\mu_{0} ; T\right)}(z)$ for any $T(\in \mathscr{Y})$ ? If it is true, is the function $\chi^{\left(\mu_{0} ; T\right)}(z)=\bar{\chi}^{\left(\mu_{0} ; T\right)}(z)=\underline{\chi}^{\left(\mu_{0} ; T\right)}(z)$ identically equal 
to some constant in $D_{r}$ ? If it is also true, what is the constant? It is conjectured that this constant will be equal to 1 .

17. ExAmple. We gave an example of Kleinian groups with fundamental domains bounded by four circles whose singular sets have positive 1-dimensional measure ([3]). Using the result of Theorem 2, we shall find the more precise property about the singular set of a Kleinian group.

Consider the three circles $H_{j}(j=1,2,3)$ with centers $a_{j}=2 e^{i(4 j-1) \pi / 6}$ $\left(j=1,2,3 ; i^{2}=-1\right)$ and equal radii $\sqrt{3}-\varepsilon$, respectively. We let these three circles $H_{j}(j=1,2,3)$ correspond to the elliptic transformations $T_{j}(j=1,2,3)$ with period 2. Then we obtain a Fuchsian group $G_{1}$ of the second kind with fixed circle $|z|=1+\varepsilon_{1}$, where $\varepsilon_{1}$ is determined depending only on $\varepsilon$. The singular set of $G_{1}$ is on the circle $|z|=1+\varepsilon_{1}$ and is nowhere dense. Next we describe a circle $H_{4}$ with center at the origin and the radius $2-\sqrt{3}$ and let it correspond to the elliptic transformation $T_{4}$ with period 2 .

Combining the Fuchsian group $G_{1}$ with $G_{2}$ generated by $T_{4}$ only, we obtain a Kleinian group $G$, that is, a combination group $G_{1} \cdot G_{2}$, whose fundamental domain $B$ is connected and bounded by four circles $H_{j}$ $(j=1,2,3,4)$.

For convenience of the calculation, we consider the limit case $\varepsilon=0$. Then $B$ is no more connected and the fixed circle of $G_{1}$ is $|z|=1$.

Denote by $D_{j}(j=1,2,3,4)$ the closed discs bounded by $H_{j}(j=1,2,3,4)$ and by $U$ the closed unit disc. Then the singular set $E$ of $G$ lies in the inside of $U \cap\left\{\bigcup_{j=1}^{4} D_{j}\right\}$.

By the symmetricity of the figure, it is sufficient to calculate the values of the computing functions $\chi_{n}^{\left(2 ; T_{1}\right)}(z)$ and $\chi_{n}^{\left(2 ; T_{4}\right)}(z)$ of order $n$ in $U \cap D_{1}$ and $D_{4}$, respectively.

In the case of order $n=1,2,3,4$, we can not obtain the inequality $\chi_{n}^{\left(2: T_{1}\right)}(z)>1$. But in the case of order $n=5$, we get the result which satisfies the condition of Proposition 4 in the following (see [3] with respect to the calculation):

$$
\chi_{5}^{\left(2: T_{1}\right)}(z)>1.002004, \quad \chi_{5}^{\left(2: T_{4}\right)}(z)>2.218873 .
$$

By using the method in the proof of Lemma 2, we can find that the 1-dimensional measure $M_{1}(E)$ of the singular set $E$ of this group $G$ is infinite.

18. Application of Theorem 2. Here we shall give the application of Theorem 2. Let $H(z)$ be a rational function none of whose poles is contained in the singular set $E$ of the Kleinian group $G$ defined in $\S 1$.

Consider the series 


$$
\Theta_{\mu}(z)=\sum_{j=0}^{\infty} H\left(z_{j}\right)\left(c_{j} z+d_{j}\right)^{-\mu},
$$

where $\mu$ is a positive integer and the summation is taken over all elements $z_{j}=\left(a_{j} z+b_{j}\right) /\left(c_{j} z+d_{j}\right)$ of $G$, in particular, $z_{0}$ means the identity transformation. This is a so-called $(-\mu)$-dimensional Poincare theta-series.

We put $P_{\mu}(z)=\sum_{j=0}^{\infty}\left|c_{j} z+d_{j}\right|^{-\mu}$, where $\mu$ is a positive number. We call $P_{\mu}(z)$ the $(-\mu)$-dimensional $P$-series. We have the following theorem (see [2] as to the proof).

Theorem 5 ([2]). Let $\mu$ be a positive number. The following three propositions are equivalent to each other: (i) The (- $\mu)$-dimensional $P$ series $P_{\mu}(z)$ converges uniformly in any compact domain not containing the suitable neighborhoods of the poles of $P_{\mu}(z)$. (ii) The series $\sum_{j=1}^{\infty}\left|c_{j}\right|^{-\mu}$ converges. (iii) The series $\sum_{m=1}^{\infty} l_{m}^{(\mu)}$ converges, where $l_{m}^{(k)}$ is the sum of terms $\left(r^{(m-1)}\right)^{\mu / 2}$ obtained for radii $r^{(m-1)}$ of all circles of grade $m-1$.

In particular, if $\mu$ is a positive integer, the following proposition is also equivalent to each of the above propositions: The $(-\mu)$-dimensional Poincare theta-series $\Theta_{\mu}(z)$ coverges absolutely and uniformly in any compact domain not containing the suitable neighborhoods of the poles of $\Theta_{\mu}(z)$.

It is evident that, if $\lim _{m \rightarrow \infty} l_{m}^{(\mu)}=0$, then the singular set of $G$ is of $(\mu / 2)$-dimensional measure zero. Hence, from the above theorem, we get the following result.

COROLlARY. If any one of the conditions (i), (ii) and (iii) in Theorem 5 is valid, then $M_{\mu / 2}(E)=0$.

19. The converse of the above corollary. Now let us suppose that $M_{\mu / 2}(E)=0$. Then from Theorem 2, for any sufficiently small $\varepsilon$ there exists some positive integer $n_{0}$ such that it holds

$$
\chi_{n_{0}}^{(\mu, T)}(z)<\varepsilon
$$

for any $T(\in \mathscr{Y})$ and any $z \in E \cap D_{T}$. If we determine the grade number $l_{0}$ depending only on the above $\varepsilon$ in the same way as the proof of Lemma 5 , it holds

$$
\chi_{n_{0}}^{(\mu ; T)}\left(S_{(m)}(\infty)\right)<\varepsilon
$$

for all images $S_{(m)}(\infty)$ of the infinity which are contained in $N(N-1)^{m-1}$ closed discs bounded by circles of grade $m\left(\geqq l_{0}\right)$. Hence from (1.8) and (3.4) we have for any transformation $S_{(m)}$ with grade $m\left(\geqq l_{0}\right)$

$$
\sum_{S_{\left(n_{0}\right)}}\left(R_{S_{\left(n_{0}\right)} S_{(m)}}\right)^{\mu}<\varepsilon\left(R_{S_{(m)}}\right)^{\mu} .
$$


Denote by $L_{m}^{(\mu)}$ the sum of terms $\left(R_{S_{(m)}}\right)^{\mu}$ obtained for radii $R_{S_{(m)}}$ of all isometric circles of grade $m$, that is,

$$
L_{m}^{(\mu)}=\sum_{i=1}^{N(N-1)}{ }^{m-1}\left(R_{S_{(m}^{(i)}}^{(i)}\right)^{\mu}
$$

Then the sum $\sum_{j=1}^{\infty}\left|c_{j}\right|^{-\mu}$ is written in the following way:

$$
\sum_{j=1}^{\infty}\left|c_{j}\right|^{-\mu}=\sum_{m=1}^{\infty} L_{m}^{(\mu)}=\sum_{m=1}^{\infty} \sum_{i=1}^{N(N-1)^{m-1}}\left(R_{S_{(m)}^{(i)}}\right)^{\mu} .
$$

Therefore in order to show the convergence of the series $\sum_{j=1}^{\infty}\left|c_{j}\right|^{-\mu}$, it is enough to show the convergence of the series

$$
\sum_{m=l_{0}}^{\infty} L_{m}^{(\mu)}
$$

where $l_{0}$ is the grade number determined by the above $\varepsilon$. Then we get from (3.5)

$$
\sum_{m=l_{0}}^{\infty} L_{m}^{(\mu)}<\left(\sum_{m=l_{0}}^{l_{0}+\left(n_{0}-1\right)} L_{m}^{(\mu)}\right) \sum_{t=0}^{\infty} \varepsilon^{t}=\frac{1}{1-\varepsilon} \sum_{m=l_{0}}^{l_{0}+\left(n_{0}-1\right)} L_{m}^{(\mu)}
$$

Thus we could prove the convergence of the series $\sum_{j=1}^{\infty}\left|c_{j}\right|^{-\mu}$ under the assumption $M_{\mu / 2}(E)=0$.

Thus we obtain from the above corollary the following theorem.

THEOREM 6. Let $\mu$ be a positive number. Three propositions in Theorem 5 and $M_{\mu / 2}(E)=0$ are equivalent to each other.

\section{REFERENCES}

[1] L. V. Ahlfors, Fundamental polyhedrons and limit point set of Kleinian groups. Proc. of Nat. Acad. Sci., 55 (1966), 251-254.

[2] T. AKAzA, Poincaré theta-series and singular sets of Schottky groups. Nagoya Math. J., 24 (1964), 43-65.

[3] T. AKAzA, Singular sets of some Kleinian groups (II). Ibid., 29 (1967), 145-162.

[4] A. F. BEARDON, The Hausdorff dimension of singular set of properly discontinuous groups. Amer. J. Math., 88 (1966), 722-736.

[5] L. R. Ford, Automorphic functions. 2nd Ed. Chelsea, New York. (1951).

[6] F. Sсноттку, Über eine specielle Funktion, welche bei einer bestimmten linearen Transformation ihres Arguments unverändert bleibt. Crelle's J. Math., 101 (1887), 227-272.

Department of Mathematics

KANAZAWA UNIVERSITY

AND

HARVARD UNIVERSITY 